\title{
Novel finite point approach for solving time-fractional convection-dominated diffusion equations
}

\author{
Xiaomin Liu', Muhammad Abbas²* ${ }^{2 *}$, Honghong Yang ${ }^{3}$, Xinqiang Qin ${ }^{3}$ and Tahir Nazir ${ }^{2}$
}

\section{"Correspondence:}

muhammad.abbas@uos.edu.pk

${ }^{2}$ Department of Mathematics,

University of Sargodha, 40100

Sargodha, Pakistan

Full list of author information is

available at the end of the article

\section{Springer}

\begin{abstract}
In this paper, a stabilized numerical method with high accuracy is proposed to solve time-fractional singularly perturbed convection-diffusion equation with variable coefficients. The tailored finite point method (TFPM) is adopted to discrete equation in the spatial direction, while the time direction is discreted by the G-L approximation and the L1 approximation. It can effectively eliminate non-physical oscillation or excessive numerical dispersion caused by convection dominant. The stability of the scheme is verified by theoretical analysis. Finally, one-dimensional and two-dimensional numerical examples are presented to verify the efficiency of the method.
\end{abstract}

MSC: $26 \mathrm{~A} 33 ; 35 \mathrm{R} 11 ; 65 \mathrm{M} 06 ; 65 \mathrm{M} 12 ; 65 \mathrm{~F} 40 ; 65 \mathrm{~F} 50 ; 65 \mathrm{H} 10 ; 65 \mathrm{~N} 06$

Keywords: Time-fractional convection-dominated diffusion equation; Tailored finite point method; Exponential basis function; Cauchy-Schwarz inequality

\section{Introduction}

Fractional calculus is a generalization of traditional integer-order calculus to noninteger order (fractional order). The fractional integrals and derivatives are of nonlocal property because they are quasi-differential operators. So, they provide valuable tools for describing the memory and genetic properties of different materials and processes, as well as the dynamics of complex systems controlled by anomalous diffusion $[1,2]$. The fractional calculus has a long history of rapid development and widespread application [3-5], and it is involved in nonlinear oscillating earthquakes [6], hydrodynamic models [7], continuous statistical mechanics [8], physical phenomena modeling [9], colored noise [10], solid mechanics [11], economics [12], anomalous transport [13], bioengineering [14] and many other aspects. Fractional partial differential equations (FPDEs) are characterized by noninteger-order derivatives, so they can effectively describe the memory and genetic properties of matter and play important roles in engineering, physics, fluid mechanics, mathematical biology, electrochemistry, and other science. As part of the fractional dynamic equation, fractional convection-diffusion equation is a powerful tool to simulate various anomalous diffusion phenomena. Time-fractional convection-diffusion equation

(c) The Author(s) 2021. This article is licensed under a Creative Commons Attribution 4.0 International License, which permits use sharing, adaptation, distribution and reproduction in any medium or format, as long as you give appropriate credit to the original author(s) and the source, provide a link to the Creative Commons licence, and indicate if changes were made. The images or other third party material in this article are included in the article's Creative Commons licence, unless indicated otherwise in a credit line to the material. If material is not included in the article's Creative Commons licence and your intended use is not permitted by statutory regulation or exceeds the permitted use, you will need to obtain permission directly from the copyright holder. To view a copy of this licence, visit http://creativecommons.org/licenses/by/4.0/. 
can be utilized to simulate the time-related abnormal diffusion process. It is a generalization of the classical convection-diffusion equation by replacing the integer-order time derivative with a fractional-order time derivative, which is widely used in oil reservoir simulations, transport of mass and energy, dispersion of chemicals in reactors, etc. In recent decades, scholars in different fields have pointed out and confirmed that fractional model is more suitable than integer model to simulate the process of memory, genetic heterogeneity, and the abnormal power transmission. Therefore, it is of theoretical and practical significance to find the numerical solutions of FPDE. Several numerical methods have been introduced to solve FPDE, such as the finite difference method [15, 16], finite element method [17], variational iteration method [18, 19], operational method [20], Sinc-Legendre collocation method [21], generalized differential transform method [22], etc.

In the recent years, several numerical methods have been proposed for solving the timefractional convection-diffusion equation (TFCDE). Saadamandi et al. [21] used the SincLegendre collocation method for the solution of one-dimensional equation with homogeneous boundary conditions. Uddin and Haq [23] applied radial basis functions for the numerical solution of equation with constant coefficients, and Mohammad and Jafar [24] proposed a spectral method based on Gegenbauer collocation for solving this problem. In [25], the mixed generalized Jacobi and Chebyshev collocation methods were used to solve one-dimensional equations with variable coefficient. In addition, for a class of equations with variable coefficients, the third type of Chebyshev wavelet method was discussed in [26], and Cui [27] derived a compact difference scheme to solve this problem numerically. Furthermore, Wang et al. [28] proposed high-order exponential ADI format for solving two-dimensional TFCDE. Deng [29] proposed numerical algorithm for the timefractional Fokker-Planck equation. Gorenflo [30] studied time-fractional diffusion equation using discrete random walk approach. There have been extensive works of high-order accurate schemes for Caputo derivative [31-35]. Kumar et al.[36-46] gave several methods for models with fractional derivative. Agarwal [47-50] proposed some methods for other kinds of equations with fractional derivative recently. Although the above numerical methods have solved TFCDE with various conditions to some extent, few of them could consider the effect of convection dominant, which means that the diffusion coefficient $\varepsilon$ is extremely small. When the equation is convection dominant, the use of traditional numerical methods (central difference method or Galerkin method) will produce nonphysical shock or excessive numerical diffusion (upwind difference method). Therefore, it is of significant importance in developing effective numerical method for the solution of convection dominant problem.

In this paper, we use the tailored finite point method (TFPM) to solve the time-fractional convection-dominant diffusion problem with variable coefficient, and we find that this algorithm is very effective. TFPM is based on the local exponential basis function, which was first proposed by Han et al. [51] for solving the Hemker problem numerically. In many cases, TFPM can preserve the important local properties of the problem. Subsequently, Han et al. used TFPM to solve the second-order singularly perturbed elliptic equation in [52]. In [53], TFPM was proposed for solving the parabolic problem. Han and Huang applied the method to solving the fourth-order singular perturbation elliptic equation in [54]. Huang et al. [55, 56] used TFPM to solve the surface layer problem and the first-order wave equation. Moreover, Tsai et al. [57] applied it to the numerical solution 
of one-dimensional Burgers' equation. Motivated by the advantages of TFPM, we adopt TFPM discrete in the spatial direction and use the G-L and L1 approximations of Caputo derivative discrete in the time direction in this paper to solve one-dimensional and two-dimensional time-fractional convection-dominated diffusion equations numerically. The research shows that TFPM is an efficient method to solve the convection-dominated problem.

The paper is organized as follows. In Sect. 2, we adopt TFPM for the steady problems, and then we use the G-L approximation and L1 approximation for the time-fractional derivative to give a highly efficient discrete scheme for one-dimensional time-fractional convection-dominated diffusion equation. In Sect. 3, we solve two-dimensional timefractional convection-dominated diffusion equation numerically. In Sect. 4, we theoretically analyze the stability of the method in this paper. Finally, numerical examples of one dimension and two dimensions are given respectively in Sect. 5 to verify the high efficiency of the proposed algorithm. This paper closes with a short summary in Sect. 6.

\section{One-dimensional time fractional convection-dominated diffusion equation}

Let us consider the following time-fractional convection-diffusion equation:

$$
\frac{\partial^{\gamma} u(x, t)}{\partial t^{\gamma}}-\varepsilon \frac{\partial^{2} u(x, t)}{\partial x^{2}}+p(x, t) \frac{\partial u(x, t)}{\partial x}=f(x, t), \quad x \in I, t \in(0, T]
$$

where $\varepsilon$ is the diffusion coefficient, $0<\varepsilon \ll 1, p(x, t) \neq 0$ is a continuous function; $f$ is the source term; $I=\left(L_{1}, L_{2}\right)$ is the calculation interval, $\partial I$ is the boundary; the fractional derivative $\frac{\partial^{\gamma} u(x, t)}{\partial t^{\gamma}}$ is the Caputo fractional derivative ${ }_{0}^{c} D_{t}^{\gamma} u(0<\gamma<1)$ of the function $u(x, t)$. The derivative is ${ }_{0}^{c} D_{t}^{\gamma} u=\frac{1}{\Gamma(1-\gamma)} \int_{0}^{t} \frac{\partial u(x, t)}{\partial \tau} \cdot \frac{1}{(t-\tau)^{\gamma}} d \tau$.

Corresponding boundary conditions and initial conditions of equation (2.1) are:

$$
\begin{aligned}
& u\left(L_{1}, t\right)=\mu_{1}(t), \quad u\left(L_{2}, t\right)=\mu_{2}(t), \\
& u(x, 0)=v(x) .
\end{aligned}
$$

We assume that $\Omega=\left(L_{1}, L_{2}\right) \times(0, T]$, and we take a uniform partition, i.e., let $\tau=T / N T$ be the time step and $h=\left(L_{2}-L_{1}\right) /(N X+1)$ be the mesh size for some positive integers $N T, N X \in \mathbf{N}$. Take

$$
x_{j}=L_{1}+j h \quad(j=0,1, \ldots, N X), \quad t^{n}=n \tau \quad(n=0,1, \ldots, N T) .
$$

Then $\left\{P_{j}^{n}=\left(x_{j}, t^{n}\right), 0 \leq j \leq N X, 0 \leq n \leq N T\right\}$ is the set of mesh points.

\subsection{TFPM discretization for second-order derivative}

We take the TFPM discrete scheme on the cell $I_{j}$ (see Fig. 1)

$$
\left.u_{x x}\right|_{x=x_{j}}=\alpha_{j-1} u_{j-1}+\alpha_{j} u_{j}+\alpha_{j+1} u_{j+1},
$$

where $\alpha_{j-1}, \alpha_{j}, \alpha_{j+1}$ are satisfied with a relationship, as described below.

Figure 1 Stencil for constructing the second-order derivative

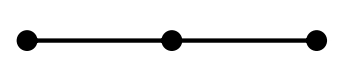


Assume that $u(x)$ can be linearly expressed by basis function on the stencil $I_{j}$, and let

$$
V=\operatorname{span}\left\{e^{-x / \varepsilon}, e^{x / \varepsilon}\right\}
$$

with $u_{j}=u\left(x_{j}, t\right)$, such that it holds for all $u \in V$ on $I_{j}$. Thus we obtain

$$
\left.u(x)\right|_{I_{j}}=c_{1 j} e^{-\left(x-x_{j}\right) / \varepsilon}+c_{2 j} e^{\left(x-x_{j}\right) / \varepsilon} .
$$

Taking (2.6) in $\alpha_{j-1} u_{j-1}+\alpha_{j} u_{j}+\alpha_{j+1} u_{j+1}=0$, we obtain

$$
\alpha_{j-1}\left[c_{1 j} e^{-h / \varepsilon}+c_{2 j} e^{h / \varepsilon}\right]+\alpha_{j}\left[c_{1 j}+c_{2 j}\right]+\alpha_{j+1}\left[c_{1 j} e^{h / \varepsilon}+c_{2 j} e^{-h / \varepsilon}\right]=0 .
$$

Then we obtain

$$
\left\{\begin{array}{l}
\alpha_{j-1} e^{-h / \varepsilon}+\alpha_{j}+\alpha_{j+1} e^{h / \varepsilon}=0 \\
\alpha_{j-1} e^{h / \varepsilon}+\alpha_{j}+\alpha_{j+1} e^{-h / \varepsilon}=0 .
\end{array}\right.
$$

Solving the above linear system, we get

$$
\alpha_{j-1}=\alpha_{j+1}=-\frac{\alpha_{j}}{e^{h / \varepsilon}+e^{-h / \varepsilon}} .
$$

Then we obtain the TFPM scheme as follows:

$$
\left.u_{x x}\right|_{x=x_{j}}=-\frac{\alpha_{j}}{e^{h / \varepsilon}+e^{-h / \varepsilon}} u_{j-1}+\alpha_{j} u_{j}-\frac{\alpha_{j}}{e^{h / \varepsilon}+e^{-h / \varepsilon}} u_{j+1},
$$

where $\alpha_{j}$ satisfies the discrete maximum principle.

\subsection{TFPM for one-dimensional time-fractional convection-dominated diffusion equation}

2.2.1 TFPM based on G-L approximation

For equation (2.1), we apply the TFPM discrete in the spatial direction and adopt the G-L approximation discrete in the temporal direction. First, we give the definition of shifted G-L derivative as follows:

$$
{ }_{0}^{G} D_{t}^{\gamma} u(t)=\tau^{-\gamma} \sum_{k=0}^{n} w_{k}^{(\gamma)} u_{j}^{n-k},
$$

where $w_{k}^{(\gamma)}=(-1)^{j}\left(\begin{array}{c}\gamma \\ j\end{array}\right), j=0,1,2, \ldots$. The discretization scheme of TFPM based on the G-L approximation for equation (2.1) is

$$
\left\{\begin{array}{l}
\tau^{-\gamma} \sum_{k=0}^{n} w_{k}^{(\gamma)} u_{j}^{n-k}=\varepsilon \alpha_{j-1} u_{j-1}^{n}+\varepsilon \alpha_{j} u_{j}^{n}+\varepsilon \alpha_{j+1} u_{j+1}^{n}+p_{j}^{n} \frac{u_{j+1}^{n}-u_{j-1}^{n}}{2 h}+f_{j}^{n}, \\
u_{j}^{0}=v(x), \quad 0 \leq j \leq N X, \\
u_{0}^{n}=\mu_{1}(t), \quad u_{N X}^{n}=\mu_{2}(t), \quad 0 \leq n \leq N T .
\end{array}\right.
$$

Let $u^{n}=\left(u_{1}^{n}, u_{2}^{n}, \ldots, u_{N X-1}^{n}\right)^{T}$, then (2.12) can be rewritten in the following matrix form:

$$
\tau^{-\gamma} \sum_{k=0}^{\infty} w_{k}^{(\gamma)} u_{j}^{n-k}=A u^{n}+F^{n},
$$


where $\alpha_{j-1}, \alpha_{j}, \alpha_{j+1}$ are determined by (2.9), and

$$
\begin{aligned}
& A=\operatorname{diag}\left(\varepsilon \alpha_{j-1}-\frac{p_{j}^{n}}{2 h}, \varepsilon \alpha_{j}, \varepsilon \alpha_{j+1}+\frac{p_{j}^{n}}{2 h}\right)_{(N X-1) \times(N X-1)}, \\
& F^{n}=\left(f_{1}^{n}+\frac{u_{0}^{n}}{\varepsilon \alpha_{j-1}}, \ldots, f_{N X}^{n}+\frac{u_{N X}^{n}}{\varepsilon \alpha_{j+1}}\right)^{T} .
\end{aligned}
$$

\subsubsection{TFPM based on L1 approximation}

For equation (2.1), we apply the TFPM discrete in the spatial direction and adopt the L1 approximation discrete in the temporal direction.

According to the definition of Caputo fractional derivative,

$$
\left.{ }_{0}^{c} D_{t}^{\gamma} u(t)\right|_{t=t_{n}}=\frac{1}{\Gamma(1-\gamma)} \int_{0}^{t_{n}} \frac{u^{\prime}(s)}{\left(t_{n}-s\right)^{\gamma}} d s=\frac{1}{\Gamma(1-\gamma)} \sum_{k=1}^{n} \int_{t_{k-1}}^{t_{k}} \frac{u^{\prime}(s)}{\left(t_{n}-s\right)^{\gamma}} d s
$$

The linear interpolation of $u(s)$ on the $\left[t_{k-1}, t_{k}\right]$ interval is obtained.

$$
\begin{aligned}
& L_{1, k}(s)=\frac{t_{k}-s}{\tau} u\left(t_{k-1}\right)+\frac{s-t_{k-1}}{\tau} u\left(t_{k}\right), \\
& u(s)-L_{1, k}(s)=\frac{1}{2} u^{\prime \prime}\left(\xi_{k}\right)\left(s-t_{k-1}\right)\left(s-t_{k}\right), \quad s \in\left[t_{k-1}, t_{k}\right],
\end{aligned}
$$

where

$$
\xi_{k}=\xi_{k}(s) \in\left(t_{k-1}, t_{k}\right) .
$$

$L_{1, k u}(s)$ approximate $u(s)$ to get

$$
\begin{gathered}
u(s) \approx \frac{t_{k}-s}{\tau} u\left(t_{k-1}\right)+\frac{s-t_{k-1}}{\tau} u\left(t_{k}\right) \\
\Rightarrow u^{\prime}(s) \approx \frac{u\left(t_{k}\right)-u\left(t_{k-1}\right)}{\tau} .
\end{gathered}
$$

And then

$$
\begin{aligned}
\left.{ }_{0}^{c} D_{t}^{\gamma} u(t)\right|_{t=t_{n}} & \frac{1}{\Gamma(1-\gamma)} \sum_{k=1}^{n} \int_{t_{k-1}}^{t_{k}} \frac{u\left(t_{k}\right)-u\left(t_{k-1}\right)}{\tau} \cdot \frac{1}{\left(t_{n}-s\right)^{\gamma}} d s \\
= & \frac{1}{\Gamma(1-\gamma)} \sum_{k=1}^{n} \frac{u\left(t_{k}\right)-u\left(t_{k-1}\right)}{\tau} \cdot \int_{t_{k-1}}^{t_{k}} \frac{1}{\left(t_{n}-s\right)^{\gamma}} d s \\
= & \frac{1}{\Gamma(1-\gamma)} \sum_{k=1}^{n} \frac{u\left(t_{k}\right)-u\left(t_{k-1}\right)}{\tau} \cdot \frac{1}{1-\gamma} \\
& \cdot\left[\left(t_{n}-t_{k-1}\right)^{1-\gamma}-\left(t_{n}-t_{k}\right)^{1-\gamma}\right]
\end{aligned}
$$




$$
\begin{aligned}
& =\frac{\tau^{-\gamma}}{\Gamma(2-\gamma)} \sum_{k=1}^{n} a_{n-k}^{(\gamma)}\left[u\left(t_{k}\right)-u\left(t_{k-1}\right)\right] \\
& =\frac{\tau^{-\gamma}}{\Gamma(2-\gamma)}\left[a_{0}^{(\gamma)} u\left(t_{n}\right)-\sum_{k=1}^{n-1}\left(a_{n-k-1}^{(\gamma)}-a_{n-k}^{(\gamma)}\right) u\left(t_{k}\right)-a_{n-1}^{(\gamma)} u\left(t_{0}\right)\right] .
\end{aligned}
$$

Then the discretization scheme of TFPM based on the L1 approximation for equation (2.1) is

$$
\left\{\begin{array}{l}
\frac{\tau^{\gamma}}{\Gamma(2-\gamma)}\left[a_{0}^{(\gamma)} u_{j}^{n}-\sum_{k=1}^{n-1}\left(a_{n-k-1}^{(\gamma)}-a_{n-k}^{(\gamma)}\right) u_{j}^{k}-a_{n-1}^{(\gamma)} u_{j}^{0}\right] \\
\quad=\varepsilon \alpha_{j-1} u_{j-1}^{n}+\varepsilon \alpha_{j} u_{j}^{n}+\varepsilon \alpha_{j+1} u_{j+1}^{n}+p_{j}^{n} \frac{u_{j+1}^{n}-u_{j-1}^{n}}{2 h}+f_{j}^{n}, \\
u_{j}^{0}=v(x), \quad 0 \leq j \leq N X, \\
u_{0}^{n}=\mu_{1}(t), \quad u_{N X}^{n}=\mu_{2}(t), \quad 0 \leq n \leq N T .
\end{array}\right.
$$

Rewrite (2.21) as the following matrix form:

$$
\frac{\tau^{\gamma}}{\Gamma(2-\gamma)}\left[a_{0}^{(\gamma)} u_{j}^{n}-\sum_{k=1}^{n-1}\left(a_{n-k-1}^{(\gamma)}-a_{n-k}^{(\gamma)}\right) u_{j}^{k}-a_{n-1}^{(\gamma)} u_{j}^{0}\right]=A u^{n}+F^{n}
$$

where $A, F^{n}$ are defined in (2.22) and $\alpha_{j-1}, \alpha_{j}, \alpha_{j+1}$ are determined by (2.9).

\section{Two-dimensional time-fractional convection-dominated diffusion equation}

Let us consider the following time-fractional convection-diffusion equation:

$$
\left\{\begin{array}{l}
{ }_{0}^{c} D_{t}^{\gamma} u(x, y, t)-\varepsilon^{2} \Delta u+p(x, y, t) u_{x}+q(x, y, t) u_{y} \\
\quad=\hat{f}(x, y, t), \quad(x, y) \in \Omega, t>0 \\
u(x, y, 0)=\mu_{0}(t), \quad(x, y) \in \Omega \\
u(x, y, t)=\mu_{1}(t), \quad(x, y) \in \partial \Omega, t>0
\end{array}\right.
$$

where $\varepsilon$ is the diffusion coefficient. $\Omega$ is a bounded area, $\partial \Omega$ is a smooth boundary; $p(x, y, t) \neq 0, q(x, y, t) \neq 0$ are continuous functions; $\hat{f}$ is the source term.

Rewrite the above equation as follows:

$$
{ }_{0}^{c} D_{t}^{\gamma} u(x, y, t)-\varepsilon^{2} \Delta u+p(x, y, t) u_{x}+q(x, y, t) u_{y}=\bar{f}+f=\hat{f}(x, y, t)
$$

where $\bar{f}(x, y, t)$ is the source term for the time variable and $f(x, y, t)$ is the source term for the spatial variable.

We assume that $\Omega=\left[x_{L}, x_{R}\right] \times\left[y_{L}, y_{R}\right], t \in(0, T]$, and we take a uniform partition, i.e., let $h_{x}=h_{y}=h$ be the mesh size and $\tau=\Delta t$ be the time step, and

$$
x_{i}=i h_{x}, \quad y_{j}=j h_{y}, \quad t_{n}=n \tau, \quad 0 \leq i \leq N X, 0 \leq j \leq N Y, 0 \leq n \leq N T .
$$

Then $\left\{P_{i, j}^{k}=\left(x_{i}, y_{j}, t_{n}\right), 0 \leq i \leq N X, 0 \leq j \leq N Y, 0 \leq n \leq N T\right\}$ is the set of mesh points. 
Figure 2 The local mesh for spatial discrete

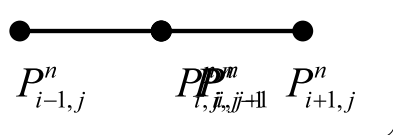

\subsection{TFPM discretization in spatial direction}

For equation (3.2), let

$$
p_{i, j}^{n}=p\left(x_{i}, y_{j}, t_{n}\right), \quad q_{i, j}^{n}=q\left(x_{i}, y_{j}, t_{n}\right), \quad f_{i, j}^{n}=f\left(x_{i}, y_{j}, t_{n}\right) .
$$

Then the equation corresponding to the spatial direction of equation (3.2) is

$$
-\varepsilon^{2} \Delta u+p_{i, j}^{n} u_{x}+q_{i, j}^{n} u_{y}=f_{i, j}^{n}
$$

We now construct our tailored finite point scheme for (3.5) on cell $\Omega_{0}$ (see Fig. 2).

Let

$$
u(x, y, t)=v(x, y, t) e^{\frac{p_{i, j}^{n} x+q_{i, j}^{n} y}{2 \varepsilon^{n}}}+\frac{p_{i, j}^{n} x+q_{i, j}^{n} y}{p_{i, j}^{n 2}+q_{i, j}^{n 2}} f_{i, j}^{n} .
$$

Substituting the above formula to (3.5), we can obtain the following equation:

$$
-\varepsilon^{2} \Delta u+d_{i, j}^{n 2} u=0
$$

where $d_{i, j}^{n 2}=\frac{p_{i, j}^{n 2}+q_{i, j}^{n 2}}{4 \varepsilon^{2}}$.

Let $\mu_{i, j}^{n}=\frac{d_{i, j}^{n}}{\varepsilon}$, and let the base function space be as follows:

$$
H_{4}=\left\{v(x, y, t) \mid v=c_{1} e^{-\mu_{i, j}^{n} x}+c_{2} e^{\mu_{i, j}^{n} x}+c_{3} e^{-\mu_{i, j}^{n} y}+c_{4} e^{\mu_{i, j}^{n} y}, \forall c_{i} \in R\right\} .
$$

We take the scheme as follows (see Fig. 2):

$$
\alpha_{1} V_{1}+\alpha_{2} V_{2}+\alpha_{3} V_{3}+\alpha_{4} V_{4}+\alpha_{0} V_{0}=0
$$

where $V_{0}=v\left(x_{i}, y_{j}, t_{n}\right), \quad V_{1}=v\left(x_{i+1}, y_{j}, t_{n}\right), \quad V_{2}=v\left(x_{i}, y_{j+1}, t_{n}\right), \quad V_{3}=v\left(x_{i-1}, y_{j}, t_{n}\right), \quad V_{4}=$ $v\left(x_{i}, y_{j-1}, t_{n}\right)$. Due to $\alpha_{k} \in R(k=0,1,2,3,4)$, so that it holds for all $v \in H_{4}$. Thus we obtain

$$
\begin{aligned}
& \alpha_{1} e^{-\mu_{i, j}^{n} h}+\alpha_{2}+\alpha_{3} e^{\mu_{i, j}^{n} h}+\alpha_{4}+\alpha_{0}=0, \\
& \alpha_{1} e^{\mu_{i, j}^{n} h}+\alpha_{2}+\alpha_{3} e^{-\mu_{i, j}^{n} h}+\alpha_{4}+\alpha_{0}=0, \\
& \alpha_{1}+\alpha_{2} e^{-\mu_{i, j}^{n} h}+\alpha_{3}+\alpha_{4} e^{\mu_{i, j}^{n} h}+\alpha_{0}=0, \\
& \alpha_{1}+\alpha_{2} e^{\mu_{i, j}^{n} h}+\alpha_{3}+\alpha_{4} e^{-\mu_{i, j}^{n} h}+\alpha_{0}=0 .
\end{aligned}
$$

Solving the above linear system (3.10)-(3.13), we get

$$
\alpha_{1}=\alpha_{2}=\alpha_{3}=\alpha_{4}=\frac{-\alpha_{0}}{e^{\mu_{i, j}^{n} h}+e^{-\mu_{i, j}^{n} h}+2}=\frac{-\alpha_{0}}{4 \cosh ^{2}\left(\frac{\mu_{i, j}^{n} h}{2}\right)} .
$$


Let

$$
\alpha_{0}=\frac{e^{\mu_{i, j}^{n} h}+e^{-\mu_{i, j}^{n} h}+2}{e^{\mu_{i, j}^{n} h}+e^{-\mu_{i, j}^{n} h}-2}=\frac{\cosh ^{2}\left(\frac{\mu_{i, j}^{n} h}{2}\right)}{\sinh ^{2}\left(\frac{\mu_{i, j}^{n} h}{2}\right)} .
$$

We can obtain

$$
\alpha_{1}=\alpha_{2}=\alpha_{3}=\alpha_{4}=-\frac{1}{4 \sinh ^{2}\left(\frac{\mu_{i, j}^{n}}{2}\right)} .
$$

Finally, we obtain the discrete scheme of equation (3.5) as follows:

$$
\begin{gathered}
u_{i, j}^{n}-\frac{e^{-\frac{p_{i, j}^{n} h}{2 \varepsilon^{2}}} u_{i+1, j}^{n}+e^{-\frac{q_{i, j}^{n} h}{2 \varepsilon^{2}}} u_{i, j+1}^{n}+e^{\frac{p_{i, j}^{n} h}{2 \varepsilon^{2}}} u_{i-1, j}^{n}+e^{\frac{q_{i, j}^{n} h}{2 \varepsilon^{2}}} u_{i, j-1}^{n}}{4 \cosh ^{2}\left(\frac{u_{i, j}^{n} h}{2}\right)} \\
=\frac{f_{i, j}^{n} h}{8 \cosh ^{2}\left(\frac{u_{i, j}^{n} h}{2}\right)}\left(\frac{e^{\frac{p_{i, j}^{n} h}{2 \varepsilon^{2}}}-e^{-\frac{p_{i, j}^{n} h}{2 \varepsilon^{2}}}}{p_{i, j}^{n 2}}+\frac{e^{\frac{q_{i, j}^{n} h}{2 \varepsilon^{2}}}-e^{-\frac{q_{i, j}^{n} h}{2 \varepsilon^{2}}}}{q_{i, j}^{n 2}}\right) .
\end{gathered}
$$

\subsection{TFPM for two-dimensional time-fractional convection-dominated diffusion equation}

For equation (3.1), the spatial direction is discreted by TFPM, and the time direction is discreted by the G-L approximation of the Caputo fractional derivative and the L1 approximation, respectively.

\subsubsection{TFPM based on G-L approximation}

Combining equations (3.2) and (3.17), we can get the following GL-TFPM discrete scheme:

$$
\begin{gathered}
\tau^{-\gamma} \sum_{k=0}^{n} w_{k}^{(\gamma)} u_{i, j}^{n-k}+u_{i, j}^{n} \\
-\frac{e^{-\frac{p_{i, h}^{n} h}{2 \varepsilon^{2}}} u_{i+1, j}^{n}+e^{-\frac{q_{i, j}^{n} h}{2 \varepsilon^{2}}} u_{i, j+1}^{n}+e^{\frac{p_{i, j}^{n} h}{2 \varepsilon^{2}}} u_{i-1, j}^{n}+e^{\frac{q_{i, j}^{n} h}{2 \varepsilon^{2}}} u_{i, j-1}^{n}}{4 \cosh ^{2}\left(\frac{u_{i, j}^{n}}{2}\right)} \\
=\frac{f_{i, j}^{n} h}{8 \cosh ^{2}\left(\frac{u_{i, j}^{n} h}{2}\right)}\left(\frac{e^{\frac{p_{i, j}^{n} h}{2 \varepsilon^{2}}}-e^{-\frac{p_{i, j}^{n} h}{2 \varepsilon^{2}}}}{p_{i, j}^{n 2}}+\frac{e^{\frac{q_{i, j}^{n} h}{2 \varepsilon^{2}}}-e^{-\frac{q_{i, j}^{n} h}{2 \varepsilon^{2}}}}{q_{i, j}^{n 2}}\right)+\bar{f}_{i, j}^{n}=\hat{f}_{i, j}^{n},
\end{gathered}
$$

where $\mu_{i, j}^{n}=\frac{d_{i, j}^{n}}{\varepsilon}, d_{i, j}^{n 2}=\frac{p_{i, j}^{n 2}+q_{i, j}^{n 2}}{4 \varepsilon^{2}}$. 


\subsubsection{TFPM based on L1 approximation}

Combining equations (3.2) and (3.17), we can get the following L1-TFPM discrete scheme:

$$
\begin{gathered}
\frac{\tau^{\gamma}}{\Gamma(2-\gamma)}\left[a_{0}^{(\gamma)} u_{i, j}^{n}-\sum_{k=1}^{n-1}\left(a_{n-k-1}^{(\gamma)}-a_{n-k}^{(\gamma)}\right) u_{i, j}^{k}-a_{n-1}^{(\gamma)} u_{i, j}^{0}\right] \\
+u_{i, j}^{n}-\frac{e^{-\frac{p_{i, j}^{n} h}{2 \varepsilon^{2}}} u_{i+1, j}^{n}+e^{-\frac{q_{i, j}^{n} h}{2 \varepsilon^{2}}} u_{i, j+1}^{n}+e^{\frac{p_{i, j}^{n} h}{2 \varepsilon^{2}}} u_{i-1, j}^{n}+e^{\frac{q_{i, j}^{n} h}{2 \varepsilon^{2}}} u_{i, j-1}^{n}}{4 \cosh ^{2}\left(\frac{u_{i, j}^{n} h}{2}\right)} \\
=\frac{f_{i, j}^{n} h}{8 \cosh ^{2}\left(\frac{u_{i, j}^{n} h}{2}\right)}\left(\frac{e^{\frac{p_{i, j}^{n} h}{2 \varepsilon^{2}}}-e^{-\frac{p_{i, j}^{n} h}{2 \varepsilon^{2}}}}{p_{i, j}^{n 2}}+\frac{e^{\frac{q_{i, j}^{n} h}{2 \varepsilon^{2}}}-e^{-\frac{q_{i, j}^{n} h}{2 \varepsilon^{2}}}}{q_{i, j}^{n 2}}\right)+\bar{f}_{i, j}^{n}=\hat{f}_{i, j}^{n},
\end{gathered}
$$

where $\mu_{i, j}^{n}=\frac{d_{i, j}^{n}}{\varepsilon}, d_{i, j}^{n 2}=\frac{p_{i, j}^{n 2}+q_{i, j}^{n 2}}{4 \varepsilon^{2}}$.

\section{Stability analysis}

\subsection{Stability analysis for one-dimensional time-fractional convection-dominated diffusion equation}

4.1.1 Stability analysis of TFPM based on G-L approximation

Theorem 1 Assume that $\left\{v_{j}^{n} \mid 0 \leq j \leq N X, 0 \leq n \leq N T\right\}$ is the solution of the GL-TFPM discrete scheme of (2.12) as $v_{0}^{n}=0, v_{N X}^{n}=0,0 \leq n \leq N T$. Then we have

$$
\left\|v^{n}\right\|_{\infty} \leq \frac{5}{1-\gamma}\left\|v^{0}\right\|_{\infty}+\frac{5}{(1-\gamma) 2^{\gamma}} t_{n}^{\gamma} \max _{1 \leq m \leq n}\left\|f^{m}\right\|_{\infty}, \quad 1 \leq n \leq N T
$$

where $\left\|f^{m}\right\|_{\infty}=\max _{1 \leq j \leq N X-1}\left|f_{j}^{m}\right|$.

Proof Rewrite equation (4.1) as follows:

$$
\begin{aligned}
& \left(1-\alpha_{j}\right) v_{j}^{n}=\sum_{k=1}^{n}\left(-w_{k}^{(\gamma)}\right) v_{j}^{n-k}+\left(\alpha_{j-1}-\frac{1}{2 h}\right) v_{j-1}^{n}+\left(\alpha_{j+1}+\frac{1}{2 h}\right) v_{j+1}^{n}+\tau^{\gamma} f_{j}^{n}, \\
& 1 \leq j \leq N X-1, \quad 1 \leq n \leq N T .
\end{aligned}
$$

Assume that $\left\|v^{n}\right\|_{\infty}=\left|v_{j_{n}}^{n}\right|$, where $j_{n} \in\{1,2, \ldots, N X-1\}$. Let $j=j_{n}$ in (4.2), and take the absolute value in the above formula. Then the triangular inequality is used. We have

$$
\left(1-\alpha_{j}\right)\left\|v^{n}\right\|_{\infty} \leq \sum_{k=1}^{n}\left(-w_{k}^{(\gamma)}\right)\left\|v^{n-k}\right\|_{\infty}+\left(\alpha_{j-1}\left\|v^{n}\right\|_{\infty}+\alpha_{j+1}\left\|v^{n}\right\|_{\infty}\right)+\tau^{\gamma}\left\|f^{n}\right\|_{\infty}
$$

Due to $\alpha_{j-1}, \alpha_{j}, \alpha_{j+1}$ being defined by (2.9), and applying the triangular inequality, we have

$$
\left\|v^{n}\right\|_{\infty} \leq \sum_{k=1}^{n}\left(-w_{k}^{(\gamma)}\right)\left\|v^{n-k}\right\|_{\infty}+\tau^{\gamma}\left\|f^{n}\right\|_{\infty}, \quad 1 \leq n \leq N T
$$

Starting from formula (4.3), the mathematical induction method is used to prove (4.1). Let

$$
A_{n}=\frac{5}{1-\gamma}\left\|\nu^{0}\right\|_{\infty}+\frac{5}{(1-\gamma) 2^{\gamma}} n^{\gamma} \tau^{\gamma} \max _{1 \leq m \leq n}\left\|f^{m}\right\|_{\infty}, \quad 1 \leq n \leq N T
$$


From (4.3), when $n=1$, we have

$$
\left\|v^{1}\right\|_{\infty} \leq\left(-w_{1}^{(\gamma)}\right)\left\|v^{0}\right\|_{\infty}+\tau^{\gamma}\left\|f^{1}\right\|_{\infty}=\gamma\left\|v^{0}\right\|_{\infty}+\tau^{\gamma}\left\|f^{1}\right\|_{\infty} \leq A_{1} .
$$

That is, (4.1) is set up for $k=1$. Assume that (4.1) is also set up for $k=1,2, \ldots, n-1(n \geq 2)$, then it can be obtained by (4.3)

$$
\begin{aligned}
\left\|v^{n}\right\|_{\infty} & \leq \sum_{k=1}^{n-1}\left(-w_{k}^{(\gamma)}\right)\left\|v^{n-k}\right\|_{\infty}+\left(-w_{n}^{(\gamma)}\right)\left\|v^{0}\right\|_{\infty}+\tau^{\gamma}\left\|f^{n}\right\|_{\infty} \\
& \leq \sum_{k=1}^{n-1}\left(-w_{k}^{(\gamma)}\right) A_{n-k}+\gamma\left(\frac{2}{n+1}\right)^{\gamma+1}\left\|v^{0}\right\|_{\infty}+\tau^{\gamma}\left\|f^{n}\right\|_{\infty} \\
& \leq \sum_{k=1}^{n-1}\left(-w_{k}^{(\gamma)}\right) A_{n}+\gamma\left(\frac{2}{n}\right)^{\gamma}\left\|v^{0}\right\|_{\infty}+\tau^{\gamma}\left\|f^{n}\right\|_{\infty} \\
& \leq\left[\sum_{k=1}^{\infty}\left(-w_{k}^{(\gamma)}\right)-\sum_{k=n}^{\infty}\left(-w_{k}^{(\gamma)}\right)\right] A_{n}+\gamma\left(\frac{2}{n}\right)^{\gamma}\left\|v^{0}\right\|_{\infty}+\tau^{\gamma}\left\|f^{n}\right\|_{\infty} \\
& \leq\left[1-\frac{1-\gamma}{5}\left(\frac{2}{n}\right)^{\gamma}\right] A_{n}+\gamma\left(\frac{2}{n}\right)^{\gamma}\left\|v^{0}\right\|_{\infty}+\tau^{\gamma}\left\|^{n}\right\|_{\infty} \\
& \leq A_{n}-\frac{1-\gamma}{5}\left(\frac{2}{n}\right)^{\gamma}\left[A_{n}-\frac{5 \gamma}{1-\gamma}\left\|v^{0}\right\|_{\infty}-\frac{5}{1-\gamma}\left(\frac{n}{2}\right)^{\gamma} \tau^{\gamma}\left\|^{n}\right\|_{\infty}\right] \\
& \leq A_{n} .
\end{aligned}
$$

The above proof process is applied to $\left(\frac{2}{n+1}\right)^{\gamma+1}<\left(\frac{2}{n}\right)^{\gamma+1} \leq\left(\frac{2}{n}\right)^{\gamma},(n \geq 2)$. Therefore, it can be concluded that (4.1) is also established for $k=n$.

\subsubsection{Stability analysis of TFPM based on L1 approximation}

Theorem 2 Assume that $\left\{v_{j}^{n} \mid 0 \leq j \leq N X, 0 \leq n \leq N T\right\}$ is the solution of L1-TFPM scheme (2.21) as $v_{j}^{0}=v\left(x_{j}\right)=0,1 \leq j \leq N X-1, v_{0}^{n}=0, v_{N X}^{n}=0,0 \leq n \leq N T$.

Then we have

$$
\left\|v^{n}\right\|_{\infty} \leq\left\|v^{0}\right\|_{\infty}+t_{n}^{\gamma} \Gamma(1-\gamma) \max _{1 \leq m \leq n}\left\|f^{m}\right\|_{\infty}, \quad 1 \leq n \leq N T,
$$

where $\left\|f^{m}\right\|_{\infty}=\max _{1 \leq j \leq N X-1}\left|f_{j}^{m}\right|$.

Proof Rewrite equation (2.21) as follows:

$$
\begin{aligned}
a_{0}^{(\gamma)} v_{j}^{n}= & \sum_{k=1}^{n-1}\left(a_{n-k-1}^{(\gamma)}-a_{n-k}^{(\gamma)}\right) v_{j}^{k}+a_{n-1}^{(\gamma)} v_{j}^{0}+\tau^{\gamma} \Gamma(2-\gamma)\left(\alpha_{j-1} v_{j-1}^{n}+\alpha_{j} v_{j}^{n}+\alpha_{j+1} v_{j+1}^{n}\right) \\
& +\tau^{\gamma} \Gamma(2-\gamma) p_{j}^{n}\left(\frac{1}{2 h} v_{j+1}^{n}-\frac{1}{2 h} v_{j-1}^{n}\right) \\
& +\tau^{\gamma} \Gamma(2-\gamma) f_{j}^{n}, \quad 1 \leq j \leq N X-1,1 \leq n \leq N T .
\end{aligned}
$$


That is,

$$
\begin{aligned}
{\left[1-\tau^{\gamma} \Gamma(2-\gamma) \alpha_{j}\right] v_{j}^{n} \leq } & \sum_{k=1}^{n-1}\left(a_{n-k-1}^{(\gamma)}-a_{n-k}^{(\gamma)}\right) v_{j}^{k}+a_{n-1}^{(\gamma)} v_{j}^{0} \\
& +\tau^{\gamma} \Gamma(2-\gamma)\left(\alpha_{j-1}-\frac{1}{2 h} p_{j}^{n}\right) v_{j-1}^{n} \\
& +\tau^{\gamma} \Gamma(2-\gamma)\left(\alpha_{j-1}+\frac{1}{2 h} p_{j}^{n}\right) v_{j+1}^{n} \\
& +\tau^{\gamma} \Gamma(2-\gamma) f_{j}^{n}, \quad 1 \leq j \leq N X-1,1 \leq n \leq N T .
\end{aligned}
$$

Assume that $\left\|v^{n}\right\|_{\infty}=\left|v_{j_{n}}^{n}\right|$, where $j_{n} \in\{1,2, \ldots, N X-1\}$. Let $j=j_{n}$, and take the absolute value in the above formula. Then the triangular inequality is used. We have

$$
\begin{aligned}
& {\left[1-\tau^{\gamma} \Gamma(2-\gamma) \alpha_{j}\right]\left\|v^{n}\right\|_{\infty}} \\
& \leq \sum_{k=1}^{n-1}\left(a_{n-k-1}^{(\gamma)}-a_{n-k}^{(\gamma)}\right)\left\|v^{k}\right\|_{\infty}+a_{n-1}^{(\gamma)}\left\|v^{0}\right\|_{\infty} \\
& \quad+2 \tau^{\gamma} \Gamma(2-\gamma) \alpha_{j+1}\|v\|_{\infty} \\
& \quad+\tau^{\gamma} \Gamma(2-\gamma) f_{j}^{n}, \quad 1 \leq j \leq N X-1,1 \leq n \leq N T .
\end{aligned}
$$

Due to $\alpha_{j-1}, \alpha_{j}, \alpha_{j+1}$ being defined by (2.9), and applying the triangular inequality, we obtain

$$
\begin{aligned}
\left\|v^{n}\right\|_{\infty} \leq & \sum_{k=1}^{n-1}\left(a_{n-k-1}^{(\gamma)}-a_{n-k}^{(\gamma)}\right)\left\|v^{k}\right\|_{\infty} \\
& +a_{n-1}^{(\gamma)}\left[\left\|v^{0}\right\|_{\infty}+\frac{\tau^{\gamma} \Gamma(2-\gamma)}{a_{n-1}^{(\gamma)}}\left\|f^{n}\right\|_{\infty}\right], \quad 1 \leq n \leq N T .
\end{aligned}
$$

Notice that

$$
\frac{\tau^{\gamma} \Gamma(2-\gamma)}{a_{n-1}^{(\gamma)}} \leq \frac{\tau^{\gamma} \Gamma(2-\gamma)}{(1-\gamma) n^{-\gamma}}=(n \tau)^{\gamma} \Gamma(1-\gamma)
$$

then we have

$$
\begin{aligned}
\left\|v^{n}\right\|_{\infty} \leq & \sum_{k=1}^{n-1}\left(a_{n-k-1}^{(\gamma)}-a_{n-k}^{(\gamma)}\right)\left\|v^{k}\right\|_{\infty} \\
& +a_{n-1}^{(\gamma)}\left(\left\|v^{0}\right\|_{\infty}+t_{n}^{\gamma} \Gamma(1-\gamma)\left\|f^{n}\right\|_{\infty}\right), \quad 1 \leq n \leq N T .
\end{aligned}
$$

We adopt the mathematical induction with equation (4.9) to prove the conclusion.

When $n=1$, we obtain

$$
a_{0}^{(\gamma)}\left|v^{1}\right| \leq\left|\nu^{0}\right|+\tau^{\gamma} \Gamma(1-\gamma)\left|f\left(t_{1}\right)\right|
$$


It is easy to know that the conclusion is established for $n=1$. Assume that it is established for $k=1,2, \ldots, n-1$, then

$$
\begin{aligned}
\left\|v^{n}\right\|_{\infty} \leq & \sum_{k=1}^{n-1}\left(a_{n-k-1}^{(\gamma)}-a_{n-k}^{(\gamma)}\right)\left[\left\|v^{0}\right\|_{\infty}+(k \tau)^{\gamma} \Gamma(1-\gamma) \max _{1 \leq m \leq k}\left\|f^{m}\right\|_{\infty}\right] \\
& +a_{n-1}^{(\gamma)}\left[\left\|v^{0}\right\|_{\infty}+t_{n}^{\gamma} \Gamma(1-\gamma)\left\|f^{n}\right\|_{\infty}\right] \\
\leq & \left\{\sum_{k=1}^{n-1}\left(a_{n-k-1}^{(\gamma)}-a_{n-k}^{(\gamma)}\right)+a_{n-1}^{(\gamma)}\right\}\left[\left\|v^{0}\right\|_{\infty}+(n \tau)^{\gamma} \Gamma(1-\gamma) \max _{1 \leq m \leq n}\left\|f^{m}\right\|_{\infty}\right], \\
& =\left\|v^{0}\right\|_{\infty}+t_{n}^{\gamma} \Gamma(1-\gamma) \max _{1 \leq m \leq n}\left\|f^{m}\right\|_{\infty} .
\end{aligned}
$$

Therefore

$$
\left\|v^{n}\right\|_{\infty} \leq\left\|v^{0}\right\|_{\infty}+t_{n}^{\gamma} \Gamma(1-\gamma) \max _{1 \leq m \leq n}\left\|f^{m}\right\|_{\infty}, \quad 1 \leq n \leq N T
$$

That is, the conclusion is established for $k=n$.

\subsection{Stability analysis for two-dimensional time-fractional convection-dominated diffusion equation}

Let $\omega=\left\{(i, j) \mid\left(x_{i}, y_{j}\right) \in \Omega\right\}, \partial \omega=\left\{(i, j) \mid\left(x_{i}, y_{j}\right) \in \partial \Omega\right\}, \bar{\omega}=\omega \cup \partial \omega$, and define the mesh function as follows:

$$
\begin{aligned}
& V_{h}=\left\{u \mid u=\left\{u_{i j} \mid(i, j) \in \bar{\omega}\right\}\right\}, \quad u \text { is the mesh function on } \Omega, \\
& V_{h}^{\circ}=\left\{u \mid u \in V_{h} \text {;if }(i, j) \in \partial \omega, \text { then } u_{i j}=0\right\} .
\end{aligned}
$$

For mesh function $v \in V_{h}$, let $\kappa=1 / 2 \cosh \left(\frac{\mu_{i j h}^{h}}{2}\right)$, and we introduce the following notation:

$$
\begin{aligned}
& \delta_{x} v_{i-\frac{1}{2}, j}=\kappa\left(\cosh ^{2}\left(\frac{\mu_{i j}^{n} h}{2}\right) v_{i j}^{n}-e^{\frac{p_{i j}^{n} h}{\varepsilon^{2}}} v_{i-1, j}^{n}\right), \\
& \delta_{x} v_{i+\frac{1}{2}, j}=\kappa\left(e^{-\frac{p_{i j}^{n} h}{2 \varepsilon^{2}}} v_{i+1, j}^{n}-\cosh ^{2}\left(\frac{\mu_{i j}^{n} h}{2}\right) v_{i j}^{n}\right), \\
& \delta_{y} v_{i, j-\frac{1}{2}}=\kappa\left(\cosh ^{2}\left(\frac{\mu_{i j}^{n} h}{2}\right) v_{i j}^{n}-e^{\frac{q_{i j h}^{n}}{2 \varepsilon^{2}}} v_{i, j-1}^{n}\right), \\
& \delta_{x} v_{i, j+\frac{1}{2}}=\kappa\left(e^{-\frac{q_{i j}^{n} h}{2 \varepsilon^{2}}} v_{i, j+1}^{n}-\cosh ^{2}\left(\frac{\mu_{i j}^{n} h}{2}\right) v_{i j}^{n}\right), \\
& \delta_{x}^{2} v_{i, j}=\kappa\left(\delta_{x} v_{i+\frac{1}{2}, j}-\delta_{x} v_{i-\frac{1}{2}, j}\right), \\
& \delta_{y}^{2} v_{i, j}=\kappa\left(\delta_{x} v_{i, j+\frac{1}{2}}-\delta_{x} v_{i, j-\frac{1}{2}}\right), \\
& \delta_{x} \delta_{y} v_{i-\frac{1}{2}, j-\frac{1}{2}}=\kappa\left(\delta_{y} v_{i, j-\frac{1}{2}}-\delta_{y} v_{i-1, j-\frac{1}{2}}\right), \\
& \delta_{x}^{2} \delta_{y}^{2} v_{i, j}=\kappa^{2}\left(\delta_{y}^{2} v_{i-1, j}-2 \delta_{y}^{2} v_{i, j}+\delta_{y}^{2} v_{i+1, j}\right) .
\end{aligned}
$$


For the mesh function $v \in V_{h}^{\circ}$, define that

$$
\begin{aligned}
& (u, v)=4 \cosh ^{2}\left(\frac{\mu_{i j}^{n} h}{2}\right) \sum_{i=1}^{N X-1} \sum_{j=1}^{N Y-1} u_{i j} v_{i j}, \quad\|u\|=\sqrt{(u, u)}, \\
& (u, v)_{1, x}=4 \cosh ^{2}\left(\frac{\mu_{i j}^{n} h}{2}\right) \sum_{i=1}^{N X-1} \sum_{j=1}^{N Y-1}\left(\delta_{x} u_{i-\frac{1}{2}, j}\right) \delta_{x} v_{i-\frac{1}{2}, j}, \quad\left\|\delta_{x} u\right\|=\sqrt{(u, u)_{1, x}}, \\
& (u, v)_{1, y}=4 \cosh ^{2}\left(\frac{\mu_{i j}^{n} h}{2}\right) \sum_{i=1}^{N X-1} \sum_{j=1}^{N Y-1}\left(\delta_{y} u_{i, j-\frac{1}{2}}\right) \delta_{y} v_{i, j-\frac{1}{2}}, \quad\left\|\delta_{y} u\right\|=\sqrt{(u, u)_{1, y}}, \\
& (u, v)_{x, y}=4 \cosh ^{2}\left(\frac{\mu_{i j}^{n} h}{2}\right) \sum_{i=1}^{N X-1} \sum_{j=1}^{N Y-1}\left(\delta_{x} \delta_{y} u_{i-\frac{1}{2}, j-\frac{1}{2}}\right)\left(\delta_{x} \delta_{y} u_{i-\frac{1}{2}, j-\frac{1}{2}}\right), \\
& \left\|\delta_{x} \delta_{y} u\right\|=\sqrt{(u, u)_{x, y}}, \\
& \left\|\nabla_{h} u\right\|=\sqrt{\left\|\delta_{x} u\right\|^{2}+\left\|\delta_{y} u\right\|^{2}}, \quad\|u\|_{\infty}=\max _{\substack{1 \leq i \leq N X \\
1 \leq j \leq N Y}}\left|u_{i j}\right| .
\end{aligned}
$$

It is easy to verify, for any mesh function $u, v \in V_{h}^{\circ}$, we have

$$
\begin{aligned}
& \left(-\delta_{x}^{2} u, v\right):=4 \cosh ^{2}\left(\frac{\mu_{i j}^{n} h}{2}\right) \sum_{i=1}^{N X-1} \sum_{j=1}^{N Y-1}\left(-\delta_{x}^{2} u_{i, j}\right) v_{i, j}=(u, v)_{1, x} \\
& \left(-\delta_{y}^{2} u, v\right):=4 \cosh ^{2}\left(\frac{\mu_{i j}^{n} h}{2}\right) \sum_{i=1}^{N X-1} \sum_{j=1}^{N Y-1}\left(-\delta_{y}^{2} u_{i, j}\right) v_{i, j}=(u, v)_{1, y} \\
& \left(\delta_{x}^{2} \delta_{y}^{2} u, v\right):=4 \cosh ^{2}\left(\frac{\mu_{i j}^{n} h}{2}\right) \sum_{i=1}^{N X-1} \sum_{j=1}^{N Y-1}\left(\delta_{x}^{2} \delta_{y}^{2} u_{i, j}\right) v_{i, j}=(u, v)_{x, y} .
\end{aligned}
$$

Lemma $1([58])$ For any $u \in V_{h}^{\circ}$, let $L_{x}=x_{R}-x_{L}, L_{y}=y_{R}-y_{L}$, then

$$
\|u\|^{2} \leq \frac{1}{\frac{6}{L_{x}^{2}}+\frac{6}{L_{y}^{2}}}\left\|\nabla_{h} u\right\|^{2} .
$$

Here, $\|\cdot\|$ indicates the $L_{2}$ norm.

\subsubsection{Stability analysis of TFPM based on G-L approximation}

Theorem 3 Assume that $\left\{v_{i j}^{n} \mid(i, j) \in \bar{\omega}, 0 \leq n \leq N T\right\}$ is the solution of GL-TFPM scheme (3.18) or as below:

$$
\begin{aligned}
& \tau^{-\gamma} \sum_{k=0}^{n} w_{k}^{(\gamma)} v_{i, j}^{n-k}=\delta_{x}^{2} v_{i j}^{n}+\delta_{y}^{2} v_{i j}^{n}+\hat{f}_{i, j}^{n}, \quad(i, j) \in \omega, 1 \leq n \leq N T, \\
& v_{i j}^{0}=\mu_{0}\left(x_{i}, y_{j}\right), \quad(i, j) \in \omega, \\
& v_{i j}^{n}=0,(i, j) \in \partial \omega, \quad 0 \leq n \leq N T .
\end{aligned}
$$


Then we have

$$
\begin{aligned}
\left\|v^{n}\right\|^{2} \leq & \frac{5}{1-\gamma}\left\|v^{0}\right\|^{2} \\
& +\frac{L_{x}^{2} L_{y}^{2}}{12\left(L_{x}^{2}+L_{y}^{2}\right)} \frac{5}{(1-\gamma) 2^{\gamma}} t_{n}^{\gamma} \max _{1 \leq m \leq n}\left\|f^{m}\right\|^{2}, \quad 1 \leq n \leq N T,
\end{aligned}
$$

where $\left\|f^{m}\right\|^{2}=4 \cosh ^{2}\left(\frac{\mu_{i j}^{n} h}{2}\right) \sum_{i=1}^{N X-1} \sum_{j=1}^{N Y-1}\left(f_{i j}^{m}\right)^{2}$.

Proof We use the inner product simultaneously on both sides of equation (4.10). Noticing (4.12) and applying (4.7)-(4.9), we can obtain

$$
\begin{aligned}
\tau^{-\gamma} \sum_{k=0}^{n} w_{k}^{(\gamma)}\left(v^{n-k}, v^{n}\right) & =-\left(v^{n}, v^{n}\right)_{1, x}-\left(v^{n}, v^{n}\right)_{1, y}+\left(\hat{f}^{n}, v^{n}\right) \\
& =-\left\|\nabla_{h} v^{n}\right\|^{2}+\left(\hat{f}^{n}, v^{n}\right), \quad 1 \leq n \leq N T .
\end{aligned}
$$

By the Cauchy-Schwarz inequality, and noting Lemma 1, we have

$$
\begin{aligned}
\left(\hat{f}^{n}, v^{n}\right) & \leq\left\|\hat{f}^{n}\right\|\left\|v^{n}\right\| \\
& \leq 6\left(\frac{1}{L_{x}^{2}}+\frac{1}{L_{y}^{2}}\right)\left\|v^{n}\right\|^{2}+\frac{1}{24\left(1 / L_{x}^{2}+1 / L_{y}^{2}\right)}\left\|\hat{f}^{n}\right\|^{2} \\
& \leq\left\|\nabla_{h} v^{n}\right\|^{2}+\frac{1}{24\left(1 / L_{x}^{2}+1 / L_{y}^{2}\right)}\left\|\hat{f}^{n}\right\|^{2}, \quad 1 \leq n \leq N T .
\end{aligned}
$$

Combining (4.15) and (4.14), we can get

$$
\tau^{-\gamma} \sum_{k=0}^{n} w_{k}^{(\gamma)}\left(v^{n-k}, v^{n}\right) \leq \frac{1}{24\left(1 / L_{x}^{2}+1 / L_{y}^{2}\right)}\left\|\hat{f}^{n}\right\|^{2}, \quad 1 \leq n \leq N T .
$$

Reorganizing the above formula and using the Cauchy-Schwarz inequality, we have

$$
\begin{aligned}
\left\|v^{n}\right\|^{2} \leq & \sum_{k=1}^{n}\left(-w_{k}^{(\gamma)}\right)\left(v^{n-k}, v^{n}\right)+\frac{L_{x}^{2} L_{y}^{2}}{24\left(L_{x}^{2}+L_{y}^{2}\right)} \tau^{\gamma}\left\|\hat{f}^{n}\right\|^{2} \\
\leq & \sum_{k=1}^{n}\left(-w_{k}^{(\gamma)}\right)\left[\frac{1}{2}\left(\left\|v^{n-k}\right\|^{2}+\left\|v^{n}\right\|^{2}\right)\right] \\
& +\frac{L_{x}^{2} L_{y}^{2}}{24\left(L_{x}^{2}+L_{y}^{2}\right)} \tau^{\gamma}\left\|\hat{f}^{n}\right\|^{2}, \quad 1 \leq n \leq N T .
\end{aligned}
$$

Notice that $\sum_{k=1}^{n}\left(-w_{k}^{(\gamma)}\right) \leq w_{0}^{(\gamma)}=1$, and multiply by 2 on both sides of the above formula, then

$$
\left\|v^{n}\right\|^{2} \leq \sum_{k=1}^{n}\left(-w_{k}^{(\gamma)}\right)\left\|v^{n-k}\right\|^{2}+\frac{L_{x}^{2} L_{y}^{2}}{12\left(L_{x}^{2}+L_{y}^{2}\right)} \tau^{\gamma}\left\|\hat{f}^{n}\right\|^{2}, \quad 1 \leq n \leq N T .
$$

From inequality (4.16), it is easy to verify (4.13) using the mathematical induction method (similar to the induction process in Theorem 1, omitted here). 


\subsubsection{Stability analysis of TFPM based on L1 approximation}

Theorem 4 Assume that $\left\{v_{i j}^{n} \mid(i, j) \in \bar{\omega}, 0 \leq n \leq N T\right\}$ is the solution of L1-TFPM discrete scheme (3.19) or as below:

$$
\begin{aligned}
& \frac{\tau^{\gamma}}{\Gamma(2-\gamma)}\left[a_{0}^{(\gamma)} u_{i, j}^{n}-\sum_{k=1}^{n-1}\left(a_{n-k-1}^{(\gamma)}-a_{n-k}^{(\gamma)}\right) u_{i, j}^{k}-a_{n-1}^{(\gamma)} u_{i, j}^{0}\right] \\
& \quad=\delta_{x}^{2} \nu_{i j}^{n}+\delta_{y}^{2} v_{i j}^{n}+\hat{f}_{i, j}^{n}, \quad(i, j) \in \omega, 1 \leq n \leq N T,
\end{aligned}
$$

where

$$
v_{i j}^{0}=\mu_{0}\left(x_{i}, y_{j}\right), \quad(i, j) \in \omega,
$$

and

$$
v_{i j}^{n}=0, \quad(i, j) \in \partial \omega, 0 \leq n \leq N T .
$$

Then we have

$$
\left\|v^{n}\right\|^{2} \leq\left\|v^{0}\right\|^{2}+\frac{L_{x}^{2} L_{x}^{2}}{12\left(L_{x}^{2}+L_{y}^{2}\right)} \Gamma(1-\gamma) t_{n}^{\gamma} \max _{1 \leq m \leq n}\left\|f^{m}\right\|^{2}, \quad 1 \leq n \leq N T
$$

where $\left\|f^{m}\right\|^{2}=4 \cosh ^{2}\left(\frac{\mu_{i j}^{n} h}{2}\right) \sum_{i=1}^{N X-1} \sum_{j=1}^{N Y-1}\left(f_{i j}^{m}\right)^{2}$.

Proof Taking both sides of equation (4.17) with $v^{n}$ for the inner product $(\cdot, \cdot)$ simultaneously, we have

$$
\begin{aligned}
& \frac{\tau^{\gamma}}{\Gamma(2-\gamma)}\left(\left[a_{0}^{(\gamma)} v^{n}-\sum_{k=1}^{n-1}\left(a_{n-k-1}^{(\gamma)}-a_{n-k}^{(\gamma)}\right) v^{k}-a_{n-1}^{(\gamma)} v^{0}\right], v^{n}\right) \\
& =\left(\delta_{x}^{2} v^{n}, v^{n}\right)+\left(\delta_{y}^{2} v^{n}, v^{n}\right)+\left(\hat{f}^{n}, v^{n}\right), \quad 1 \leq n \leq N T .
\end{aligned}
$$

Noting (4.19) and applying (4.7)-(4.9), we can get

$$
\left(\delta_{x}^{2} v^{n}, v^{n}\right)+\left(\delta_{y}^{2} v^{n}, v^{n}\right)=-\left(v^{n}, v^{n}\right)_{1, x}-\left(v^{n}, v^{n}\right)_{1, y}=-\left\|\nabla_{h} v^{n}\right\|^{2} .
$$

By the Cauchy-Schwarz inequality and noting Lemma 1, there are

$$
\begin{aligned}
\left(\hat{f}^{n}, v^{n}\right) & \leq\left\|\hat{f}^{n}\right\|\left\|v^{n}\right\| \\
& \leq 6\left(1 / L_{x}^{2}+1 / L_{y}^{2}\right)\left\|v^{n}\right\|^{2}+\frac{1}{24\left(1 / L_{x}^{2}+1 / L_{y}^{2}\right)}\left\|\hat{f}^{n}\right\|^{2} \\
& \leq\left\|\nabla_{h} v^{n}\right\|^{2}+\frac{1}{24\left(1 / L_{x}^{2}+1 / L_{y}^{2}\right)}\left\|\hat{f}^{n}\right\|^{2}, \quad 1 \leq n \leq N T .
\end{aligned}
$$


Substituting (4.22), (4.23) into (4.21) and then applying the Cauchy-Schwarz inequality, we have

$$
\begin{aligned}
a_{0}^{(\gamma)}\left(v^{n}, v^{n}\right) \leq & \sum_{k=1}^{n-1}\left(a_{n-k-1}^{(\gamma)}-a_{n-k}^{(\gamma)}\right)\left(v^{k}, v^{n}\right)+a_{n-1}^{(\gamma)}\left(v^{0}, v^{n}\right)+\frac{\tau^{\gamma} \Gamma(2-\gamma)}{24\left(1 / L_{x}^{2}+1 / L_{y}^{2}\right)}\left\|\hat{f}^{n}\right\|^{2} \\
\leq & \frac{1}{2} \sum_{k=1}^{n-1}\left(a_{n-k-1}^{(\gamma)}-a_{n-k}^{(\gamma)}\right)\left[\left(v^{k}, v^{k}\right)+\left(v^{n}, v^{n}\right)\right] \\
& +\frac{1}{2} a_{n-1}^{(\gamma)}\left[\left(v^{0}, v^{0}\right)+\left(v^{n}, v^{n}\right)\right]+\frac{\tau^{\gamma} \Gamma(2-\gamma)}{24\left(1 / L_{x}^{2}+1 / L_{y}^{2}\right)}\left\|\hat{f}^{n}\right\|^{2} .
\end{aligned}
$$

Multiplying by 2 on both sides of the above formula, we get

$$
\begin{aligned}
a_{0}^{(\gamma)}\left(v^{n}, v^{n}\right) \leq & \sum_{k=1}^{n-1}\left(a_{n-k-1}-a_{n-k}\right)\left(v^{k}, v^{k}\right)+a_{n-1}\left(v^{0}, v^{0}\right) \\
& +\frac{\tau^{\gamma} \Gamma(2-\gamma)}{12\left(1 / L_{x}^{2}+1 / L_{y}^{2}\right)}\left\|\hat{f}^{n}\right\|^{2} .
\end{aligned}
$$

Notice that

$$
\frac{\tau^{\gamma} \Gamma(2-\gamma)}{a_{n-1}^{(\gamma)}} \leq \frac{\tau^{\gamma} \Gamma(2-\gamma)}{(1-\gamma) n^{-\gamma}}=(n \tau)^{\gamma} \Gamma(1-\gamma) .
$$

Then we obtain

$$
\begin{aligned}
\left\|v^{n}\right\|^{2} \leq & \sum_{k=1}^{n-1}\left(a_{n-k-1}-a_{n-k}\right)\left\|v^{k}\right\|^{2} \\
& +a_{n-1}^{(\gamma)}\left[\left\|v^{0}\right\|^{2}+\frac{L_{x}^{2} L_{y}^{2}}{12\left(L_{x}^{2}+L_{y}^{2}\right)} t_{n}^{\gamma} \Gamma(1-\gamma)\left\|\hat{f}^{n}\right\|^{2}\right] .
\end{aligned}
$$

From inequality (4.25), using the mathematical induction method (similar to the induction process in Theorem 2) can lead to

$$
\left\|v^{n}\right\|^{2} \leq\left\|v^{0}\right\|^{2}+\frac{L_{x}^{2} L_{y}^{2}}{12\left(L_{x}^{2}+L_{y}^{2}\right)} \Gamma(1-\gamma) t_{n}^{\gamma} \max _{1 \leq m \leq n}\left\|\hat{f}^{m}\right\|^{2}, \quad 1 \leq n \leq N T .
$$

\section{Numerical examples}

Example 1 We consider the following one-dimensional time-fractional convectiondominated diffusion equation:

$$
\left\{\begin{array}{l}
{ }_{0}^{c} D_{t}^{\gamma} u+p(x, t) u_{x}-\varepsilon u_{x x}=f(x, t), \quad 0<x<1,0<t<1, \\
u(0, t)=\mu_{1}(t), \quad u(1, t)=\mu_{2}(t), \quad 0<t<1, \\
u(x, 0)=v(x)=0, \quad 0<x<1,
\end{array}\right.
$$

where $\varepsilon$ is a nonnegative small parameter, let $\varepsilon=10^{-5}, p(x, t)=1$. The exact solution of the equation is

$$
u(x, t)=t^{2+\gamma}\left(x+\left(e^{x / \varepsilon}-1\right) /\left(e^{1 / \varepsilon}-1\right)\right) .
$$


Here, the corresponding right term, the initial conditions, and the boundary conditions can be obtained directly from the exact solution. Let the time step $\Delta t=0.01$.

There is the boundary layer on $x=1$ near the exact solution of the equation. We adopt the TFPM scheme based on G-L approximation (2.11) and the TFPM scheme based on L1 approximation (2.13) and use the classical difference scheme (DM) for numerical solution of the equation.

In order to compare the advantages and disadvantages of the algorithm in our paper, the difference scheme is calculated and the error is estimated by $L_{2}$ norm. We use the L1-TFPM discrete scheme for the equation. The results are shown in Table 3.

It can be seen from Figs. $3-5$ that the method used in this paper can effectively eliminate the numerical oscillations at the boundary layer. It can be also seen from Tables 1 and 2 that the algorithm has achieved a perfect error accuracy. The convergence rate of the L1TFPM method is presented in Table 3.

Example 2 Here we consider the following two-dimensional time-fractional convectiondominated diffusion equation:

$$
\left\{\begin{array}{l}
{ }_{0}^{c} D_{t}^{\gamma} u(x, y, t)-\varepsilon^{2} \Delta u+p(x, y, t) u_{x}+q(x, y, t) u_{y} \\
\quad=\hat{f}(x, y, t), \quad(x, y) \in \Omega, t>0 \\
u(x, y, 0)=\mu_{0}, \quad(x, y) \in \Omega, t>0 \\
u(x, y, t)=\mu_{1}, \quad(x, y) \in \partial \Omega, t>0
\end{array}\right.
$$

where $(x, y) \in \Omega=[0,1] \times[0,1]$. Let $\varepsilon^{2}=3 \times 10^{-3}, p(x, y, t)=1, q(x, y, t)=1$, the exact solution of the equation is $u(x, y, t)=t^{2+\gamma} y(1-y)\left(e^{x-1 / \varepsilon^{2}}+(x-1) e^{-1 / \varepsilon^{2}}-x\right)$, and $\hat{f}, \mu_{0} \mu_{1}$ are determined by the exact solution. Take the time step $\Delta t=0.01$.

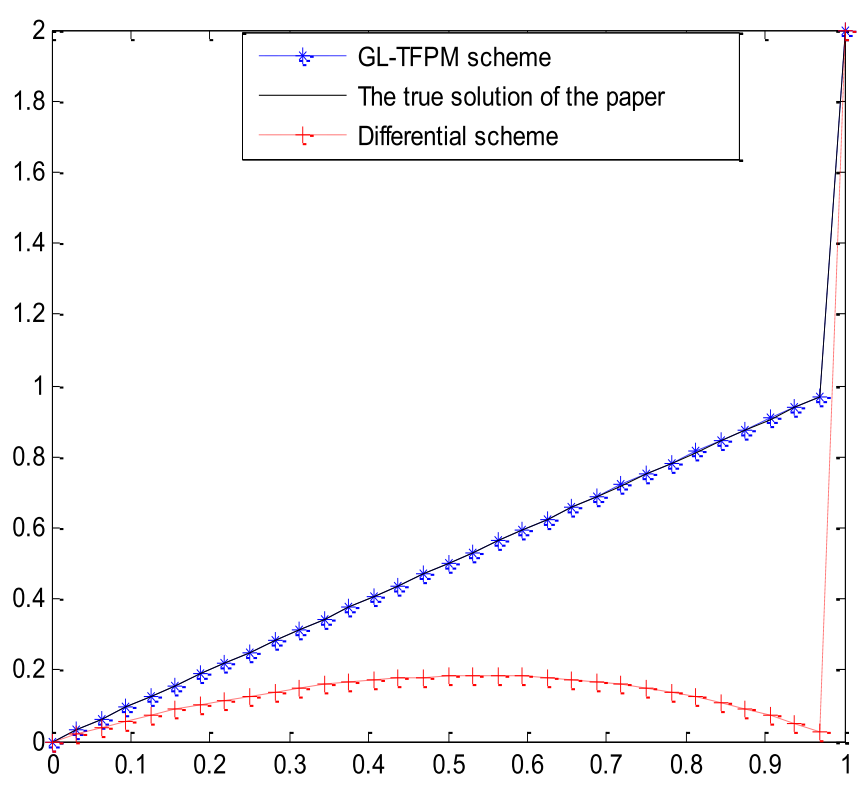

Figure 3 Comparison of GL-TFPM and DM schemes 


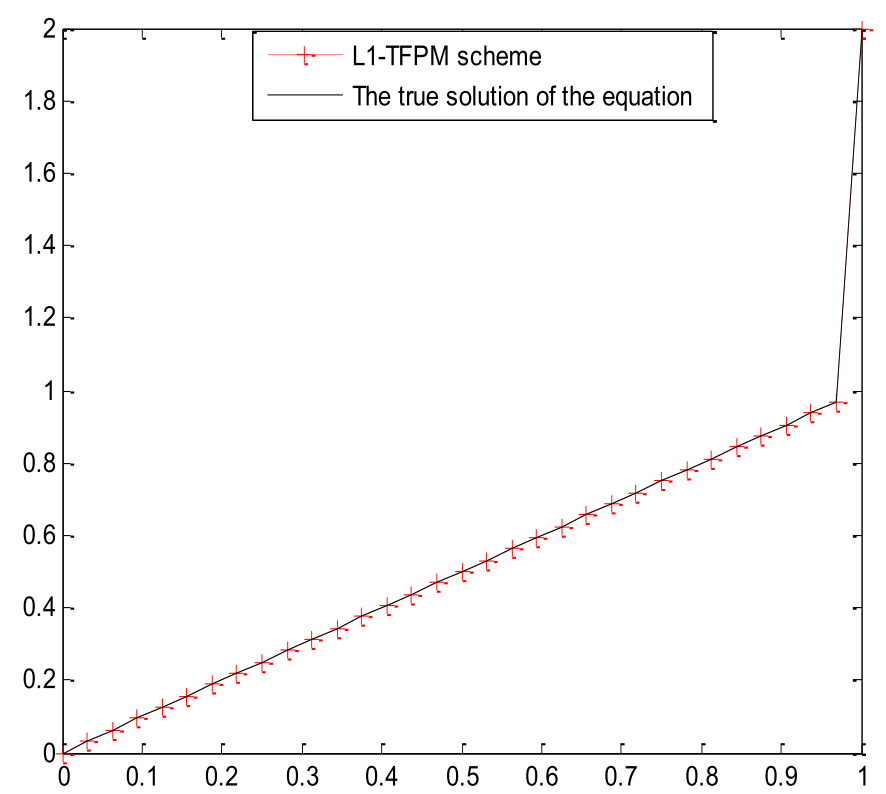

Figure 4 L1-TFPM and the exact solution

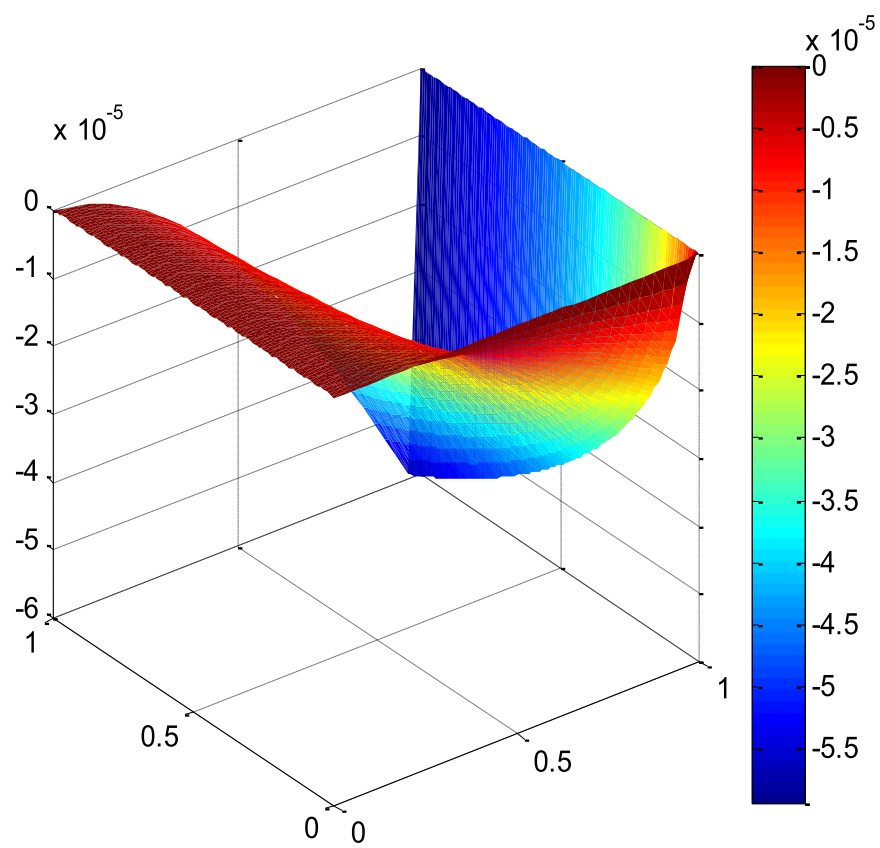

Figure 5 The GL-TFPM scheme $L^{2}$-norm error of $\gamma=0.2, h=1 / 3$

We employ the TFPM scheme based on G-L approximation (3.18) and the TFPM scheme based on L1 approximation (3.19) and use the classical difference scheme (DM) for numerical solution of the equation. Figures 6 and 7 show the three-dimensional figures of the exact solution, numerical solution of the GL-TFPM and L1-TFPM, respectively. Ta- 
Table 1 The $L^{2}$-norm error of GL-TFPM

\begin{tabular}{llllll}
\hline GL-TFPM & $N$ & $\gamma=0.1$ & $\gamma=0.3$ & $\gamma=0.5$ & $\gamma=0.8$ \\
\hline L $^{2}$-norm & 16 & $1.8741 \mathrm{e}-004$ & $6.7410 \mathrm{e}-004$ & $1.3695 \mathrm{e}-003$ & $3.0370 \mathrm{e}-004$ \\
& 32 & $1.8671 \mathrm{e}-004$ & $6.6632 \mathrm{e}-004$ & $1.3575 \mathrm{e}-003$ & $3.0239 \mathrm{e}-003$ \\
& 64 & $1.8294 \mathrm{e}-004$ & $6.5091 \mathrm{e}-004$ & $1.3270 \mathrm{e}-003$ & $2.9375 \mathrm{e}-003$ \\
& 128 & $1.7628 \mathrm{e}-004$ & $6.4179 \mathrm{e}-004$ & $1.3228 \mathrm{e}-003$ & $2.0456 \mathrm{e}-003$ \\
\hline
\end{tabular}

Table 2 Comparison of $L^{2}$-norm error between the difference scheme and the method in the paper

\begin{tabular}{lrllll}
\hline & $N$ & $\gamma=0.1$ & $\gamma=0.3$ & $\gamma=0.5$ & $\gamma=0.8$ \\
\hline L1-TFPM $\left(L^{2}\right.$-norm $)$ & 16 & $3.6293 \mathrm{e}-006$ & $2.9616 \mathrm{e}-005$ & $1.4826 \mathrm{e}-004$ & $1.9736 \mathrm{e}-003$ \\
& 32 & $3.5706 \mathrm{e}-006$ & $2.8942 \mathrm{e}-005$ & $1.4615 \mathrm{e}-004$ & $1.2638 \mathrm{e}-003$ \\
& 64 & $3.4309 \mathrm{e}-006$ & $2.8541 \mathrm{e}-005$ & $1.3479 \mathrm{e}-004$ & $1.2415 \mathrm{e}-003$ \\
& 128 & $3.2558 \mathrm{e}-006$ & $2.2658 \mathrm{e}-005$ & $1.2900 \mathrm{e}-004$ & $2.9737 \mathrm{e}-004$ \\
DM (L ${ }^{2}$-norm $)$ & 16 & $4.5172 \mathrm{e}-001$ & $7.8411 \mathrm{e}-001$ & $4.3837 \mathrm{e}-001$ & $4.1023 \mathrm{e}-001$ \\
& 32 & $4.6780 \mathrm{e}-001$ & $7.7903 \mathrm{e}-001$ & $3.5481 \mathrm{e}-001$ & $3.9876 \mathrm{e}-001$ \\
& 64 & $2.7585 \mathrm{e}-001$ & $7.7663 \mathrm{e}-001$ & $4.6309 \mathrm{e}-001$ & $4.7101 \mathrm{e}-001$ \\
& 128 & $4.7989 \mathrm{e}-001$ & $7.6865 \mathrm{e}-001$ & $1.6723 \mathrm{e}-001$ & $4.5263 \mathrm{e}-001$ \\
\hline
\end{tabular}

Table 3 The convergence order of the proposed method L1-TFPM as $\gamma=0.8$

\begin{tabular}{lll}
\hline The number of nodes & The computation error & Convergence order \\
\hline 16 & $1.9736 \mathrm{e}-003$ & \\
32 & $1.2638 \mathrm{e}-003$ & 0.64 \\
64 & $1.2415 \mathrm{e}-003$ & 0.03 \\
128 & $2.9737 \mathrm{e}-004$ & 2.06 \\
\hline
\end{tabular}
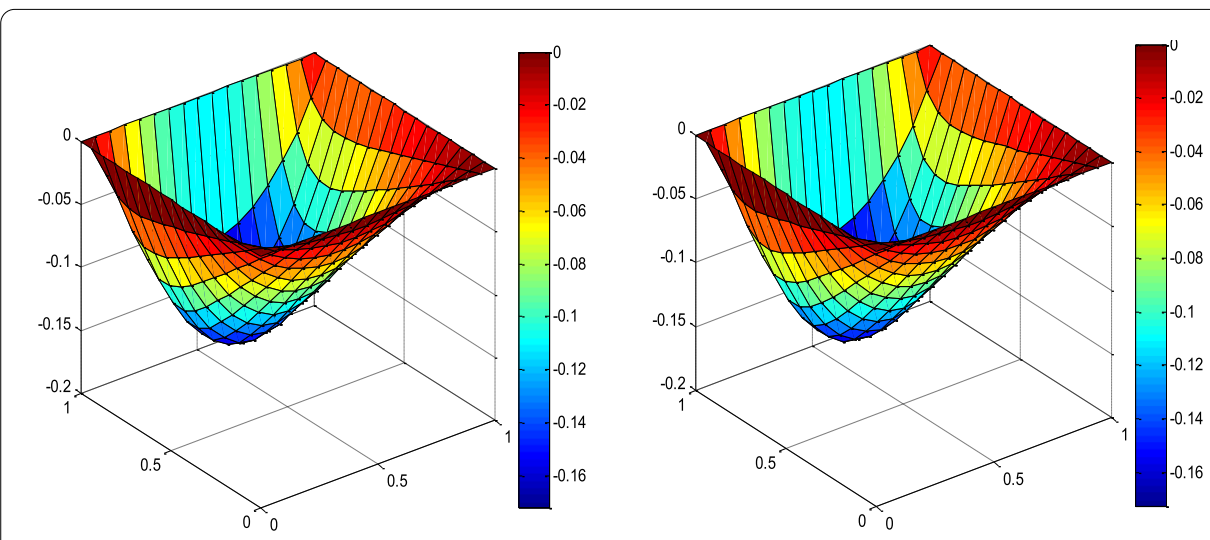

Figure 6 The exact solution and the solution of GL-TFPM of $\gamma=0.3, h x=h y=1 / 16$

ble 4 shows the error comparison between the GL-TFPM scheme and the DM scheme. Table 4 shows the error of the L1-TFPM.

As can be seen from Figs. 6-7, TFPM can effectively eliminate numerical oscillations. From Tables 4, 5 and 6, it can be also seen that the algorithm constructed in this paper is feasible and the error precision is perfectly high.

\section{Conclusion}

In this paper, the tailored finite point method to solve the time-fractional convectiondominant diffusion problem with variable coefficient is derived. And the stability based on $\mathrm{L} 1$ approximation and G-L approximation is also analyzed. At the same time, the 1D 


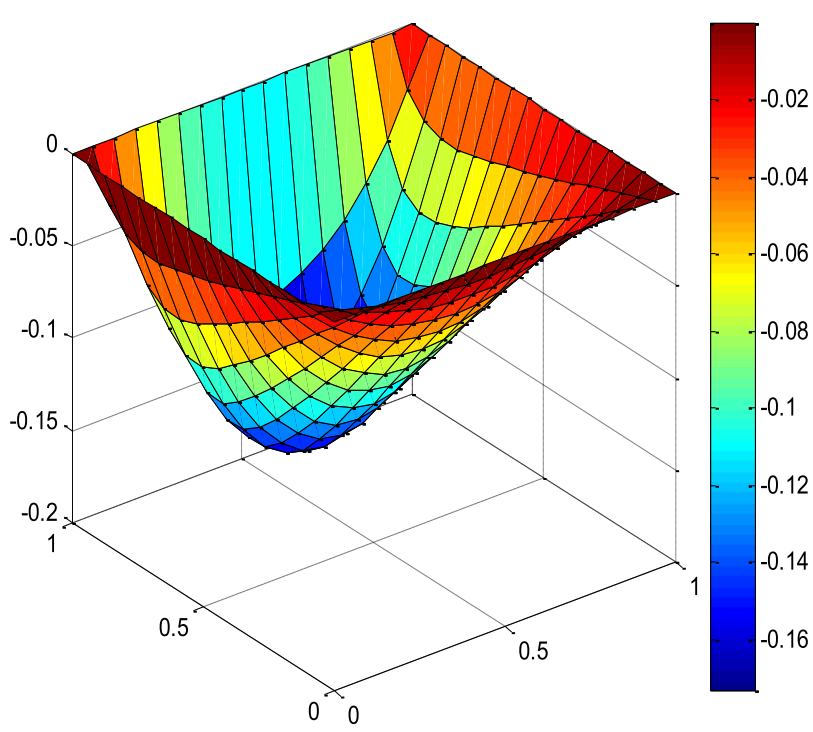

Figure 7 The solution of L1-TFPM of $\gamma=0.3, h x=h y=1 / 16$

Table 4 The comparison of error between the difference scheme and GL-TFPM

\begin{tabular}{llllll}
\hline & $N x \times N y$ & $\gamma=0.1$ & $\gamma=0.3$ & $\gamma=0.5$ & $\gamma=0.8$ \\
\hline GL-TFPM & $11 \times 11$ & $2.2629 \mathrm{e}-003$ & $3.4251 \mathrm{e}-003$ & $4.2396 \mathrm{e}-003$ & $9.3615 \mathrm{e}-003$ \\
& $21 \times 21$ & $1.0380 \mathrm{e}-003$ & $3.3749 \mathrm{e}-003$ & $6.1552 \mathrm{e}-003$ & $9.3210 \mathrm{e}-003$ \\
& $31 \times 31$ & $9.8423 \mathrm{e}-004$ & $3.1869 \mathrm{e}-003$ & $5.9977 \mathrm{e}-003$ & $9.0921 \mathrm{e}-003$ \\
& $51 \times 51$ & $7.6516 \mathrm{e}-004$ & $1.0529 \mathrm{e}-003$ & $4.0131 \mathrm{e}-003$ & $7.6581 \mathrm{e}-003$ \\
$\mathrm{DM}$ & $11 \times 11$ & $7.9724 \mathrm{e}-001$ & $7.8411 \mathrm{e}-001$ & $7.7611 \mathrm{e}-001$ & $7.4650 \mathrm{e}-001$ \\
& $21 \times 21$ & $7.9207 \mathrm{e}-001$ & $7.7903 \mathrm{e}-001$ & $7.6215 \mathrm{e}-001$ & $7.4036 \mathrm{e}-001$ \\
& $31 \times 31$ & $7.8944 \mathrm{e}-001$ & $7.7663 \mathrm{e}-001$ & $7.6186 \mathrm{e}-001$ & $7.2850 \mathrm{e}-001$ \\
& $51 \times 51$ & $7.8073 \mathrm{e}-001$ & $7.6865 \mathrm{e}-001$ & $7.5929 \mathrm{e}-001$ & $7.0866 \mathrm{e}-001$ \\
\hline
\end{tabular}

Table 5 The error of L1-TFPM in the paper

\begin{tabular}{llllll}
\hline & $N x \times N y$ & $\gamma=0.1$ & $\gamma=0.3$ & $\gamma=0.5$ & $\gamma=0.8$ \\
\hline L1-TFPM & $11 \times 11$ & $2.0811 \mathrm{e}-003$ & $7.0324 \mathrm{e}-003$ & $2.0845 \mathrm{e}-002$ & $5.1065 \mathrm{e}-002$ \\
& $21 \times 21$ & $1.2307 \mathrm{e}-003$ & $6.9693 \mathrm{e}-003$ & $3.6682 \mathrm{e}-003$ & $4.8705 \mathrm{e}-002$ \\
& $31 \times 31$ & $1.1760 \mathrm{e}-003$ & $6.7579 \mathrm{e}-003$ & $3.6122 \mathrm{e}-003$ & $4.8311 \mathrm{e}-002$ \\
& $51 \times 51$ & $9.6585 \mathrm{e}-004$ & $4.3210 \mathrm{e}-003$ & $1.8219 \mathrm{e}-003$ & $5.0135 \mathrm{e}-003$ \\
\hline
\end{tabular}

Table 6 The convergence order of the proposed method L1-TFPM as $\gamma=0.8$

\begin{tabular}{lll}
\hline The number of nodes & The computation error & Convergence order \\
\hline $11 \times 11$ & $5.1065 \mathrm{e}-002$ & \\
$21 \times 21$ & $4.8705 \mathrm{e}-002$ & 0.07 \\
$31 \times 31$ & $4.8311 \mathrm{e}-002$ & 0.02 \\
$51 \times 51$ & $5.0135 \mathrm{e}-003$ & 3.27 \\
\hline
\end{tabular}

and $2 \mathrm{D}$ cases are also numerically simulated. We compare the errors between the proposed method and the finite difference method. The numerical results show that the calculation accuracy and convergence result of the proposed method exceed DM. Therefore the tailored finite point method is an effective numerical method that can be used to solve the time-fractional convection-dominant diffusion problem. 
Acknowledgements

We thank Dr. Muhammad Kashif Iqbal for his assistance in proofreading of the manuscript.

\section{Funding}

This work is supported by the Key Research and Development Program of Shaanxi Province of China (No. 2017GY-090).

Availability of data and materials

Not applicable.

\section{Competing interests}

The authors declare that they have no competing interests.

\section{Authors' contributions}

All authors contributed equally to the writing of this paper. All authors read and approved the final manuscript.

\section{Author details}

'College of Mathematics and Computer Application, Shangluo University, Shangluo 726000, China. ${ }^{2}$ Department of Mathematics, University of Sargodha, 40100 Sargodha, Pakistan. ${ }^{3}$ Department of Applied Mathematics, Xi'an University of Technology, Xi'an 710054, China.

\section{Publisher's Note}

Springer Nature remains neutral with regard to jurisdictional claims in published maps and institutional affiliations.

\section{Received: 18 September 2020 Accepted: 13 December 2020 Published online: 06 January 2021}

\section{References}

1. Podlubny, I.: Fractional Differential Equations: An Introduction to Fractional Derivatives, Fractional Differential Equations, to Methods of Their Solution and Some of Their Applications. Elsevier, Amsterdam (1998)

2. Kilbas, A.A., Srivastava, H.M., Trujillo, J.J.: Theory and Applications of Fractional Differential Equations. Elsevier, San Diego (2006)

3. Miller, K.S., Ross, B.: An Introduction to the Fractional Calculus and Fractional Differential Equations. Wiley, New York (1993)

4. Machado, J.T., Kiryakova, V., Mainardi, F.: Recent history of fractional calculus. Commun. Nonlinear Sci. Numer. Simul. 16, 1140-1153 (2011)

5. Das, S.: Functional Fractional Calculus for System Identification and Controls. Springer, New York (2008)

6. He, J.H.: Nonlinear oscillation with fractional derivative and its applications. Int. Conf. Vib. Eng. 98, 288-291 (1998)

7. Moaddy, K., Momani, S., Hashim, I.: The non-standard finite difference scheme for linear fractional FDEs in fluid mechanics. Comput. Math. Appl. 61, 1209-1216 (2011)

8. Carpinteri, A., Mainardi, F.: Fractional Calculus: Some Basic Problems in Continuum and Statistical Mechanics, vol. 378, pp. 291-348. Springer, New York (1997)

9. Grigorenko, I., Grigorenko, E.: Chaotic dynamics of the fractional Lorenz system. Phys. Rev. Lett. 91, 034101 (2003)

10. Mandelbrot, B.: Some noises with $1 / f$ spectrum, a bridge between direct current and white noise. IEEE Trans. Inf. Theory 13, 289-298 (1967)

11. Rossikhin, Y.A., Shitikova, M.V.: Applications of fractional calculus to dynamic problems of linear and nonlinear hereditary mechanics of solids. Appl. Mech. Rev. 50, 15-67 (1997)

12. Baillie, R.T.: Long memory processes and fractional integration in econometrics. J. Econom. 73, 5-59 (1996)

13. Metzler, R., Klafter, J.: The restaurant at the end of the random walk: recent developments in the description of anomalous transport by fractional dynamics. J. Phys. 37, 161-208 (2004)

14. Magin, R.L.: Fractional calculus in bioengineering. Crit. Rev. Biomed. Eng. 32, 1-104 (2004)

15. Su, L., Wang, W., Xu, Q.: Finite difference methods for fractional dispersion equations. Appl. Math. Comput. 216, 3329-3334 (2010)

16. Murio, D.A.: Implicit finite difference approximation for time fractional diffusion equations. Comput. Math. Appl. 56 1138-1145 (2008)

17. Jiang, Y., Ma, J.: High-order finite element methods for time-fractional partial differential equations. J. Comput. Appl. Math. 235, 3285-3290 (2011)

18. Dehghan, M., Yousefi, S.A., Lotfi, A.: The use of He's variational iteration method for solving the telegraph and fractional telegraph equations. Int. J. Numer. Methods Biomed. Eng. 27, 219-231 (2011)

19. Inc, M.: The approximate and exact solutions of the space and time-fractional Burgers equations with initial conditions by variational iteration method. J. Math. Anal. Appl. 345, 476-484 (2008)

20. Luchko, Y., Gorenflo, R.: An operational method for solving fractional differential equations with the Caputo derivatives. Acta Math. Vietnam. 24, 207-233 (1999)

21. Saadatmandi, A., Dehghan, M., Azizi, M.R.: The Sinc-Legendre collocation method for a class of fractional convection-diffusion equations with variable coefficients. Commun. Nonlinear Sci. Numer. Simul. 17, 4125-4136 (2012)

22. Odibat, Z., Momani, S.: A generalized differential transform method for linear partial differential equations of fractional order. Appl. Math. Lett. 21, 194-199 (2008)

23. Uddin, M., Haq, S.: RBFs approximation method for time fractional partial differential equations. Commun. Nonlinear Sci. Numer. Simul. 16, 4208-4214 (2011)

24. Izadkhah, M.M. Saberi-Nadjafi, J.: Gegenbauer spectral method for time-fractional convection-diffusion equations with variable coefficients. Math. Methods Appl. Sci. 38, 3183-3194 (2015) 
25. Tao, S.U.N.: Mixed generalized Jacobi and Chebyshev collocation method for time-fractional convection-diffusion equations. J. Math. Res. Appl. 36, 608-620 (2016)

26. Zhou, F., Xu, X.: The third kind Chebyshev wavelets collocation method for solving the time-fractional convection diffusion equations with variable coefficients. Appl. Math. Comput. 280, 11-29 (2016)

27. Cui, M.: Compact exponential scheme for the time fractional convection-diffusion reaction equation with variable coefficients. J. Comput. Phys. 280, 143-163 (2015)

28. Wang, Z., Vong, S.: A high-order exponential ADI scheme for two dimensional time fractional convection-diffusion equations. Comput. Math. Appl. 68, 185-196 (2014)

29. Deng, W.: Numerical algorithm for the time fractional Fokker-Planck equation. J. Comput. Phys. 227, 1510-1522 (2007)

30. Gorenflo, R., Mainardi, F., Moretti, D., Paradisi, P.: Time fractional diffusion: a discrete random walk approach. Nonlinear Dyn. 29, 129-143 (2002)

31. Cao, J., Li, C., Chen, Y.: High-order approximation to Caputo derivatives and Caputo-type advection-diffusion equations (II). Fract. Calc. Appl. Anal. 18 (2015)

32. Chen, M., Deng, W.: Fourth order accurate scheme for the space fractional diffusion equations. SIAM J. Numer. Anal. $52,1418-1438(2014)$

33. Alikhanov, A.A.: A new difference scheme for the time fractional diffusion equation. J. Comput. Phys. 280, 424-438 (2015)

34. Sousa, E., Li, C.: A weighted finite difference method for the fractional diffusion equation based on the Riemann-Liouville derivative. Appl. Numer. Math. 90, 22-37 (2015)

35. LV, C., Xu, C.: Error analysis of a high order method for time-fractional diffusion equations. SIAM J. Sci. Comput. 38, A2699-A2724 (2016)

36. Kumar, S.: A new analytical modelling for fractional telegraph equation via Laplace transform. Appl. Math. Model. 38, 3154-3163 (2014)

37. Kumar, S., Rashidi, M.M.: New analytical method for gas dynamics equation arising in shock fronts. Comput. Phys. Commun. 185, 1947-1954 (2014)

38. Kumar, S., Kumar, D., Abbasbandy, S., Rashidi, M.M.: Analytical solution of fractional Navier-Stokes equation by using modified Laplace decomposition method. Ain Shams Eng. J. 5, 569-574 (2014)

39. Kumar, S., Kumar, A., Baleanu, D.: Two analytical methods for time-fractional nonlinear coupled Boussinesq-Burger's equations arise in propagation of shallow water waves. Nonlinear Dyn. 85, 699-715 (2016)

40. Kumar, S., Kumar, R., Cattani, C., Samet, B.: Chaotic behaviour of fractional predator-prey dynamical system. Chaos Solitons Fractals 135, 109811 (2020)

41. Ghanbari, B., Kumar, S., Kumar, R.: A study of behaviour for immune and tumor cells in immunogenetic tumour model with non-singular fractional derivative. Chaos Solitons Fractals 133, 109619 (2020)

42. Kumar, S., Kumar, A., Samet, B., Gómez-Aguilar, J.F., Osman, M.S.: A chaos study of tumor and effector cells in fractional tumor-immune model for cancer treatment. Chaos Solitons Fractals 141, 110321 (2020)

43. Goufo, E.F.D., Kumar, S., Mugisha, S.B.: Similarities in a fifth-order evolution equation with and with no singular kernel. Chaos Solitons Fractals 130, 109467 (2020)

44. Kumar, S., Nisar, K.S., Kumar, R., Cattani, C., Samet, B.: A new Rabotnov fractional-exponential function-based fractional derivative for diffusion equation under external force. Math. Methods Appl. Sci. 43, 4460-4471 (2020)

45. Kumar, S., Ghosh, S., Samet, B., Goufo, E.F.D.: An analysis for heat equations arises in diffusion process using new Yang-Abdel-Aty-Cattani fractional operator. Math. Methods Appl. Sci. 43, 6062-6080 (2020)

46. Kumar, S., Kumar, R., Agarwal, R.P., Samet, B.: A study of fractional Lotka-Volterra population model using Haar wavelet and Adams-Bashforth-Moulton methods. Math. Methods Appl. Sci. 43, 5564-5578 (2020)

47. Agarwal, R., Yadav, M.P., Baleanu, D., Purohit, S.D.: Existence and uniqueness of miscible flow equation through porous media with a nonsingular fractional derivative. AlMS Math. 5, 1062-1073 (2020)

48. Agarwal, R., Purohit, S.D.: Mathematical model for anomalous subdiffusion using comformable operator. Chaos Solitons Fractals 140, 110199 (2020)

49. Suthar, D.L., Purohit, S.D., Araci, S.: Solution of fractional kinetic equations associated with the (p,q)-Mathieu-type series. Discrete Dyn. Nat. Soc. 2020, 8645161 (2020)

50. Agarwal, R., Purohit, S.D.: A mathematical fractional model with nonsingular kernel for thrombin receptor activation in calcium signalling. Math. Methods Appl. Sci. 42, 7160-7171 (2019)

51. Han, H., Huang, Z., Kellogg, R.B.: The Tailored Finite Point Method and a Problem of P. Hemker. Proceedings of the International Conference on Boundary and Interior Layers Computational and Asymptotic Methods. University of Limerick, Limerick (2008)

52. Han, H., Huang, Z:: Tailored finite point method for steady-state reaction-diffusion equation. Commun. Math. Sci. 82 213-226 (2013)

53. Huang, Z., Yang, Y.: Tailored finite point method for parabolic problems. Comput. Methods Appl. Math. 16, 543-562 (2016)

54. Han, H., Huang, Z:: An equation decomposition method for the numerical solution of a fourth-order elliptic singular perturbation problem. Numer. Methods Partial Differ. Equ. 28, 942-953 (2012)

55. Huang, Z: Tailored finite point method for the interface problem. Netw. Heterog. Media 4, 91-106 (2009)

56. Huang, Z., Yang, X.: Tailored finite point method for first order wave equation. J. Sci. Comput. 49, 351-366 (2011)

57. Tsai, C.C., Shih, Y.T., Lin, Y.T., Wang, H.C.: Tailored finite point method for solving one-dimensional Burgers' equation. Int. J. Comput. Math. 94, 800-812 (2016)

58. Li, C., Zeng, F.: Finite difference methods for fractional differential equations. Int. J. Bifurc. Chaos 22, 1230014 (2012) 